\title{
BMJ Open Postpartum management of hypertensive disorders of pregnancy: a systematic review
}

\author{
Alexandra E Cairns, ${ }^{1}$ Louise Pealing, ${ }^{1}$ James M N Duffy, ${ }^{1}$ Nia Roberts, ${ }^{2}$ \\ Katherine L Tucker, ${ }^{1}$ Paul Leeson, ${ }^{3}$ Lucy H MacKillop, ${ }^{4}$ Richard J McManus ${ }^{1}$
}

To cite: Cairns AE, Pealing L, Duffy JMN, et al. Postpartum management of hypertensive disorders of pregnancy: a systematic review. BMJ Open 2017;7:e018696. doi:10.1136/ bmjopen-2017-018696

- Prepublication history and additional material for this paper are available online. To view these files, please visit the journal online (http://dx.doi. org/10.1136/bmjopen-2017018696).

Received 14 July 2017 Revised 2 October 2017 Accepted 6 October 2017

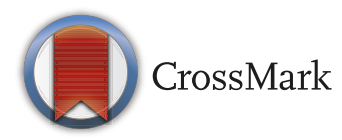

${ }^{1}$ Nuffield Department of Primary Care Health Sciences, University of Oxford, Oxford, UK

${ }^{2}$ Knowledge Centre, Bodleian Libraries, University of Oxford, Oxford, UK

${ }^{3}$ Cardiovascular Clinical Research Facility, Division of Cardiovascular Medicine, University of Oxford, Oxford, UK ${ }^{4}$ Nuffield Department of Obstetrics and Gynaecology, University of Oxford, 0xford, UK

Correspondence to

Dr Alexandra E Cairns;

alexandra.cairns@phc.ox.ac.uk

\section{ABSTRACT}

Objectives Hypertensive disorders of pregnancy (HDP) affect one in ten pregnancies and often persist postpartum when complications can occur. We aimed to determine the effectiveness and safety of pharmacological interventions, other interventions and different care models for postpartum hypertension management.

Design A systematic review was undertaken. Nine electronic databases, including Medline, were searched from inception to 16 March 2017. After duplicate removal, 4561 records were screened. Two authors independently selected studies, extracted study characteristics and data, and assessed methodological quality.

Setting Randomised controlled trials, case-control studies and cohort studies from any country and healthcare setting.

Participants Postnatal women with HDP.

Interventions Therapeutic intervention for management of hypertension, compared with another intervention, placebo or no intervention.

Primary and secondary outcome measures Outcome data were collected for maternal mortality and severe morbidity; systolic, diastolic and mean arterial blood pressure (BP) control; and safety data. Secondary outcome data collected included the length of postnatal hospital stay and laboratory values.

Results 39 studies were included $(n=2901)$. Results were heterogeneous in terms of intervention, comparison and outcome requiring a narrative approach. There were insufficient data to recommend any single pharmacological intervention. 18 studies reported calcium-channel blockers, vasodilators and beta-blockers lowered BP postpartum. 12 of these reported safety data. Limited data existed regarding management in the weeks following hospital discharge. Neither loop diuretics (three studies) nor corticosteroids (one study) produced clinical benefit. Uterine curettage significantly reduced BP over the first 48 hours postpartum (range 6-13 mm Hg) compared with standard care (eight studies), with safety data only reported by four of eight studies.

Conclusion There was insufficient evidence to recommend a particular BP threshold, agent or model of care, but three classes of antihypertensive appeared variably effective. Further comparative research, including robust safety data, is required. Curettage reduced BP, but without adequate reporting of harms, so it cannot currently be recommended.

\section{Strengths and limitations of this study}

- All types of intervention for the management of postpartum hypertension-medical, surgical and organisation of care-were eligible for inclusion in this review.

- Randomised controlled studies plus other experimental study designs (cohort studies, casecontrol studies and quasi-randomised studies) were included, and no limitations were imposed in terms of language or publication date, resulting in a comprehensive review.

- This review highlights significant evidence gaps, demonstrating that further comparative research is required, particularly to clarify postpartum antihypertensive selection.

- Although 39 studies were included, the majority had a high risk of bias such that the evidence provided by this review is of low quality.

- The 39 studies reported a broad range of heterogeneous outcomes, limiting meaningful comparison.

\section{INTRODUCTION}

Hypertensive disorders of pregnancy (HDP) often persist following delivery, ${ }^{1}$ and sometimes arise de novo postpartum. ${ }^{2}$ In both scenarios adverse events can occur during this period. Approximately one-third of eclampsia occurs postpartum, nearly half beyond 48 hours after childbirth. ${ }^{3-5}$ Half of the women who sustain an intracerebral haemorrhage in association with pre-eclampsia do so following birth. ${ }^{6}$ Women may enter the postnatal period requiring large doses of antihypertensive medication, but the majority will be treatment-free by $3-6$ months. ${ }^{17}$ This rapidly changing blood pressure (BP) poses a challenge in terms of appropriate antihypertensive selection and dose adjustment.

The National Institute for Health and Care Excellence (NICE) recommends frequent postnatal BP monitoring for women with both pre-eclampsia (every 1-2 days for 2 weeks) and gestational hypertension (at 
least once between days 3 and 5). ${ }^{8}$ The guideline stipulates thresholds for the increase or commencement $(\geq 150 / 100 \mathrm{~mm} \mathrm{Hg})$ and the reduction or cessation (consider $<140 / 90 \mathrm{~mm} \mathrm{Hg}$ and reduce $<130 / 80 \mathrm{~mm} \mathrm{Hg}$ ) of antihypertensive medication after birth. However, little detail is provided about frequency or proportion of dose reduction or how to manage multiple medications. ${ }^{8}$ The American College of Obstetricians and Gynecologists recommends that BP be monitored in hospital (or with an equivalent level of outpatient surveillance) for 72 hours after birth, and checked again 7-10 days postpartum (sooner if a woman is symptomatic).$^{9}$ In line with NICE, they propose treating BP when $\geq 150 / 100 \mathrm{~mm} \mathrm{Hg}$, but add this should be on two measures, 4-6hours apart. They make no suggestion regarding BP thresholds for medication reduction, implying uncertainty about when to decrease or stop treatment.

A Cochrane review (search date January 2013) evaluated medical interventions for prevention and treatment of postnatal hypertension. This was limited to randomised controlled trials (RCTs) and included only nine studies. ${ }^{10}$ Given the paucity of evidence available, due to Cochrane's restriction to randomised trials alone, we have undertaken an updated systematic review of the postpartum management of hypertension in women with HDP with a broader scope, including the full range of interventions studied, and incorporating cohort and case-control studies, alongside RCTs. The following were our specific questions: (1) How should BP be monitored in women with HDP postpartum? (2) What BP thresholds should be used for antihypertensive treatment initiation, adjustment and cessation postpartum? (3) Which antihypertensive medication(s) should be used in postpartum in women with HDP? (4) What are the benefits and harms of other therapeutic interventions for women with HDP postpartum?

\section{MATERIALS AND METHODS}

A protocol, with explicitly defined objectives, study selection criteria, and approaches to assessing study quality, outcomes and statistical methods, was developed (online supplementary appendix S1). This was registered with PROSPERO: International Prospective Register of Systematic Reviews (CRD42015015527) and is available online (http://www.crd.york.ac.uk/PROSPERO/ display_record.asp?ID=CRD42015015527). We followed the guidelines for meta-analyses and systematic reviews outlined by the Preferred Reporting Items for Systematic Reviews and Meta-Analyses statement (online supplementary appendix S2). ${ }^{11}$

A systematic literature review was undertaken to capture evidence from human studies regarding postpartum hypertension management in women with HDP, without restriction by language or publication date (online supplementary appendix S1). We searched the following databases, from inception to 16 March 2017: Cochrane Database of Systematic Reviews, Database of Abstracts of Reviews of Effects and Cochrane Central Register of Controlled Trials, Cumulative Index to Nursing and Allied Health Literature, Embase, Medline, PsycINFO, Science Citation Index, Science (Web of Science Core Collection), Social Science Citation Index and Conference Proceedings Citation Index. We hand-searched reference lists and contacted relevant experts for potentially relevant studies, which might have been missed by electronic searches. ${ }^{12}$

We included RCTs, quasi-randomised studies, casecontrol studies, and prospective and retrospective cohort studies assessing interventions for hypertension management postpartum in women with HDP (gestational hypertension, pre-eclampsia, chronic hypertension and superimposed pre-eclampsia) arising both during pregnancy and de novo in the postnatal period. Consistent with guidance from Cochrane, conference abstracts were included..$^{5}$

Two reviewers (AEC/LP) independently screened the titles and abstracts, and then critically reviewed the full text of selected studies to assess eligibility. Discrepancies were resolved by discussion before independent extraction of relevant data by the two reviewers. For trials with multiple intervention arms, we extracted data from eligible comparison arms. Data were extracted for the primary and secondary outcomes outlined in table 1 . Due to the heterogeneous nature of these studies, a narrative synthesis was undertaken.

Two reviewers (AEC/LP) independently assessed each trial's methodological quality using the Cochrane Collaboration's tool for assessing the risk of bias in RCTs, ${ }^{13}$ and the Newcastle-Ottawa Scale for case-control and cohort studies. ${ }^{14}$ A global assessment of bias across trials was made.

\section{RESULTS}

Our searches yielded 7105 records, and after excluding duplicates 4561 titles and abstracts were screened (figure 1). Eighty full-text articles were assessed: 35 articles were excluded (online supplementary appendix S3). Forty-five articles, representing 39 studies (32 randomised trials, 2 prospective cohort studies and 5 retrospective cohort studies) reporting data from 2901 postnatal participants, met our inclusion criteria (online supplementary appendix S4). Of the 39 studies, 9 (23\%) were published only as conference abstracts. No further details were made available following author contact.

A range of interventions were assessed, including antihypertensive medications ( 18 studies, $\mathrm{n}=982$ ), loop diuretics ( 4 studies, $n=503$ ), parenteral steroids ( 1 study, $n=157$ ), other medications ( 6 studies, $\mathrm{n}=188$ ), uterine curettage ( 8 studies, $\mathrm{n}=837$ ) and novel models of care (2 studies, $\mathrm{n}=234)$. Of the 39 studies, 9 (23\%) included $\geq 100$ participants, and only 2 studies included $\geq 200$ participants. ${ }^{1516}$ Four were from lower middle-income settings ${ }^{15}$ 17-19 (classified according to the United Nations ${ }^{20}$ ), and 13/39 (33\%) studies had follow-up periods longer than 7 days 
Table 1 Outcome measures

\section{Outcome measures}

\begin{tabular}{lll}
\hline Primary outcome(s) & Maternal mortality & \multicolumn{1}{c}{ Direct maternal deaths up } \\
& Maternal morbidity (ischaemic stroke, intracranial haemorrhage, & to postpartum; late \\
& eclamptic seizure; development of pre-eclampsia with severe features; & maternal deaths up to 1 year \\
postnatal complication requiring intervention) & postpartum \\
& Systolic blood pressure control & \\
& Diastolic blood pressure control & Mean arterial pressure control \\
& Safety data (adverse events or maternal side effects) \\
Secondary outcome(s) & Critical care admission \\
& Length of hospital stay following delivery \\
& Postnatal readmission to secondary care \\
& Antihypertensive medication requirement \\
& Urine output \\
& Laboratory values \\
& Other as defined by study
\end{tabular}

(online supplementary appendix S4). Only 5/39 (13\%) and $7 / 39(18 \%)$ studies, respectively, reported maternal mortality or major maternal morbidity, and while the majority of studies did report some measure of BP control, three did not (table 2A,B). Of the 39 studies, 19 $(49 \%)$ reported safety data (table $2 \mathrm{~A}, \mathrm{~B})$.

Of the 39 studies, 5 (13\%) studies (all evaluating antihypertensive medications) involved mixed antenatal and postnatal populations. ${ }^{17}$ 21-24 Authors were contacted to request their data set for the postnatal participants, but no data were made available. Of the 39 studies, 6 (15\%) included participants with chronic hypertension alongside women with de novo HDP (gestational hypertension or pre-eclampsia). ${ }^{22} 2325-31$ Twelve of 39 (31\%) included women with eclampsia-in one, all participants were eclamptic (online supplementary appendix S5). ${ }^{17}$

Thirty of $32(94 \%)$ included RCTs were judged to be at high overall risk of bias, by both reviewers, according to the Cochrane tool, 23/32 (72\%) for multiple domains. Only 2 of $32(6 \%)$ were thought to be clearly at low risk of bias. ${ }^{29-32}$ All included cohort studies were deemed to have a high risk of bias in at least one domain of the Newcastle-Ottawa Scale (online supplementary appendix S6).

\section{How should BP be monitored postpartum in women with HDP?} No studies specifically addressed the frequency or method of postnatal BP monitoring. Two evaluated the impact of postpartum care organisation $(n=234)$, using the postnatal readmission rate as their primary outcome (online supplementary appendix S4). Neither reported maternal mortality or morbidity, safety data nor any measure of BP control (table 2B). ${ }^{2633}$

One assessed introduction of a specialised postpartum clinic (no further details were given) and demonstrated an increased postnatal readmission and triage visit rate (22\% intervention group, 9\% control group: difference $13 \%, \mathrm{P}<0.04)$, although $86 \%$ occurred before a participant was seen in the clinic. ${ }^{33}$ The second study evaluated specialist nurse follow-up, including home visits and telephone contact, and reported no significant difference in the postnatal readmission rate compared with standard care. $^{26}$

What BP thresholds should be used for antihypertensive treatment initiation, adjustment and cessation postpartum?

No relevant studies identified.

\section{Which antihypertensive medication(s) should be used postpartum in women with HDP?}

Fourteen randomised trials $(n=645)$, one quasi-randomised trial $(n=15)$ and three retrospective cohort studies $(n=322)$ evaluated antihypertensive medications (online supplementary appendix S4). Only three studies reported maternal mortality, ${ }^{29-31} 3435$ and three reported maternal morbidity; no differences between groups were reported (table 2A). ${ }^{29-31} 3536$ Twelve studies reported safety data, in comparisons between multiple classes of antihypertensive agents (table 2A); no clear differences were established, although one study found a greater number of minor side effects reported with oral nifedipine than with oral labetalol. ${ }^{27} 28$

The vast majority of included studies evaluated either acute control of severe hypertension $(7 / 18,39 \%)$ or BP control in the few days after delivery and while women remained hospital inpatients $(8 / 18,44 \%)$. Only three studies, two published only as conference abstracts, evaluated BP control in the weeks and months following hospital discharge. ${ }^{252728} 37$

\section{Calcium-channel blockers}

Three small studies examined oral nifedipine $(n=135)$; nifedipine resulted in a greater decrease in mean arterial pressure (MAP) 18-24hours after childbirth than placebo (intervention group $93.9 \pm 1.6 \mathrm{~mm} \mathrm{Hg}$, control group 100.2 $\pm 2.6 \mathrm{~mm} \mathrm{Hg:} \mathrm{difference} 6.3 \mathrm{~mm} \mathrm{Hg}, \mathrm{P}<0.05)$, but not at other time points to 48 hours (one RCT, $n=31$ ).$^{32}$ Nifedipine controlled severe hypertension to $<160 / 100 \mathrm{~mm}$ $\mathrm{Hg}$ more quickly than labetalol (intervention group 


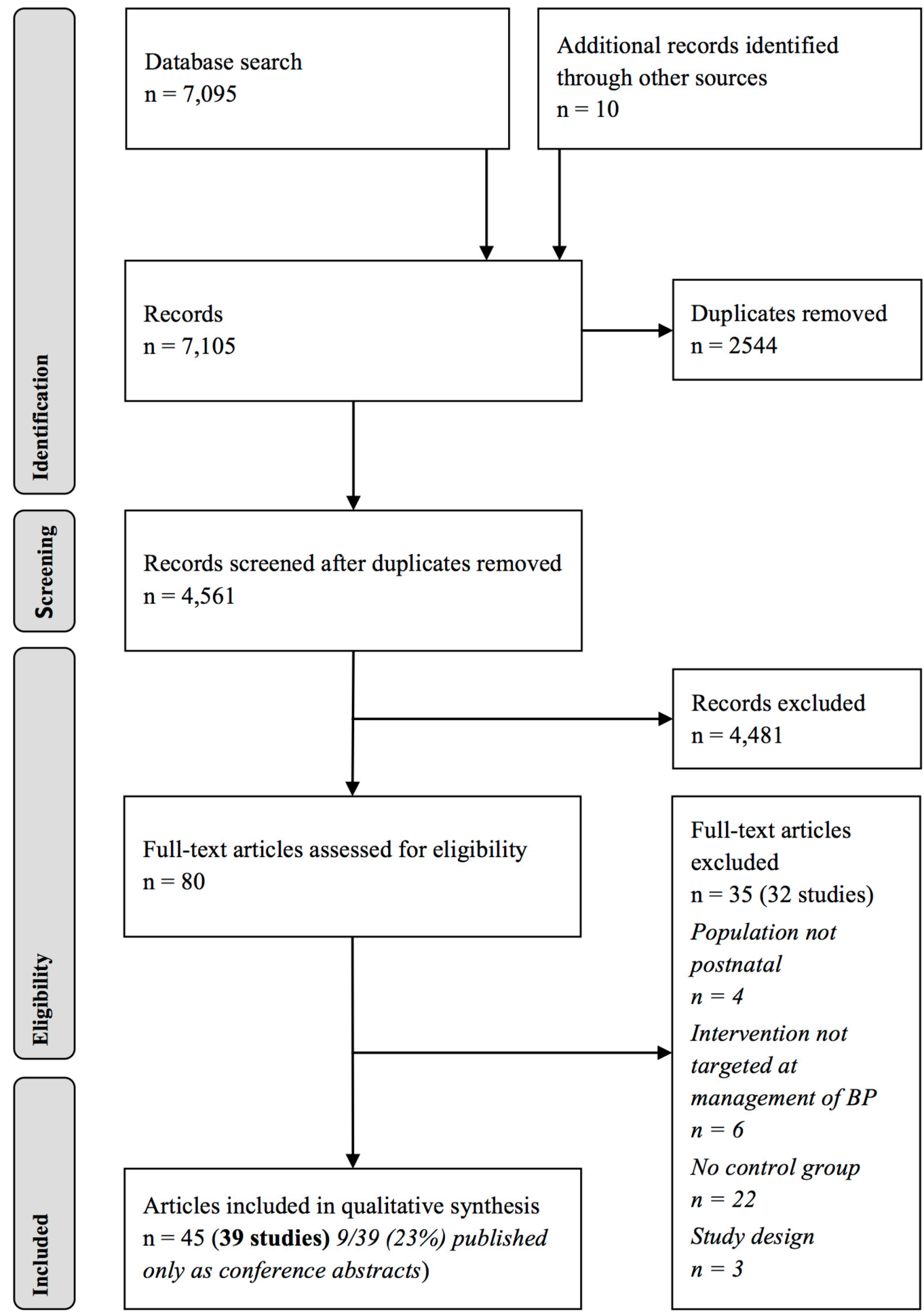

Figure 1 Preferred Reporting Items for Systematic Reviews and Meta-Analyses flow chart. BP, blood pressure.

25.1 $\pm 13.6 \mathrm{~min}$, control group 43.6 $\pm 25.4 \mathrm{~min}$ : difference 18.5 min, $\mathrm{P}=0.002$; one RCT, $\mathrm{n}=21) .^{21}$ A single RCT ( $\mathrm{n}=83$ ) reported no significant difference in time taken to control $\mathrm{BP}$ to $<150 / 100 \mathrm{~mm} \mathrm{Hg}$ when comparing nifedipine with methyldopa. ${ }^{34}$

\section{Vasodilators}

Six studies looked at the use of vasodilators $(n=252)$. All used hydralazine via a range of administration routes.
Bolus intravenous hydralazine controlled severe hypertension more quickly than continuous infusion (intervention group $65.23 \pm 23.38 \mathrm{~min}$, control group $186.36 \pm 79.77 \mathrm{~min}$ : difference $-121.13 \mathrm{~min}, \mathrm{P}<0.001$; one quasi-randomised study, $\mathrm{n}=15$ (postnatal)). ${ }^{17}$ Intramuscular hydralazine produced a more significant improvement in MAP at 6 hours than intravenous methyldopa (intervention group $104.5 \mathrm{~mm} \mathrm{Hg}$, control group $112 \mathrm{~mm} \mathrm{Hg:} \mathrm{differ-}$ ence $-7.5 \mathrm{~mm} \mathrm{Hg}, \mathrm{P}=0.0057$ ) but not at other time points 


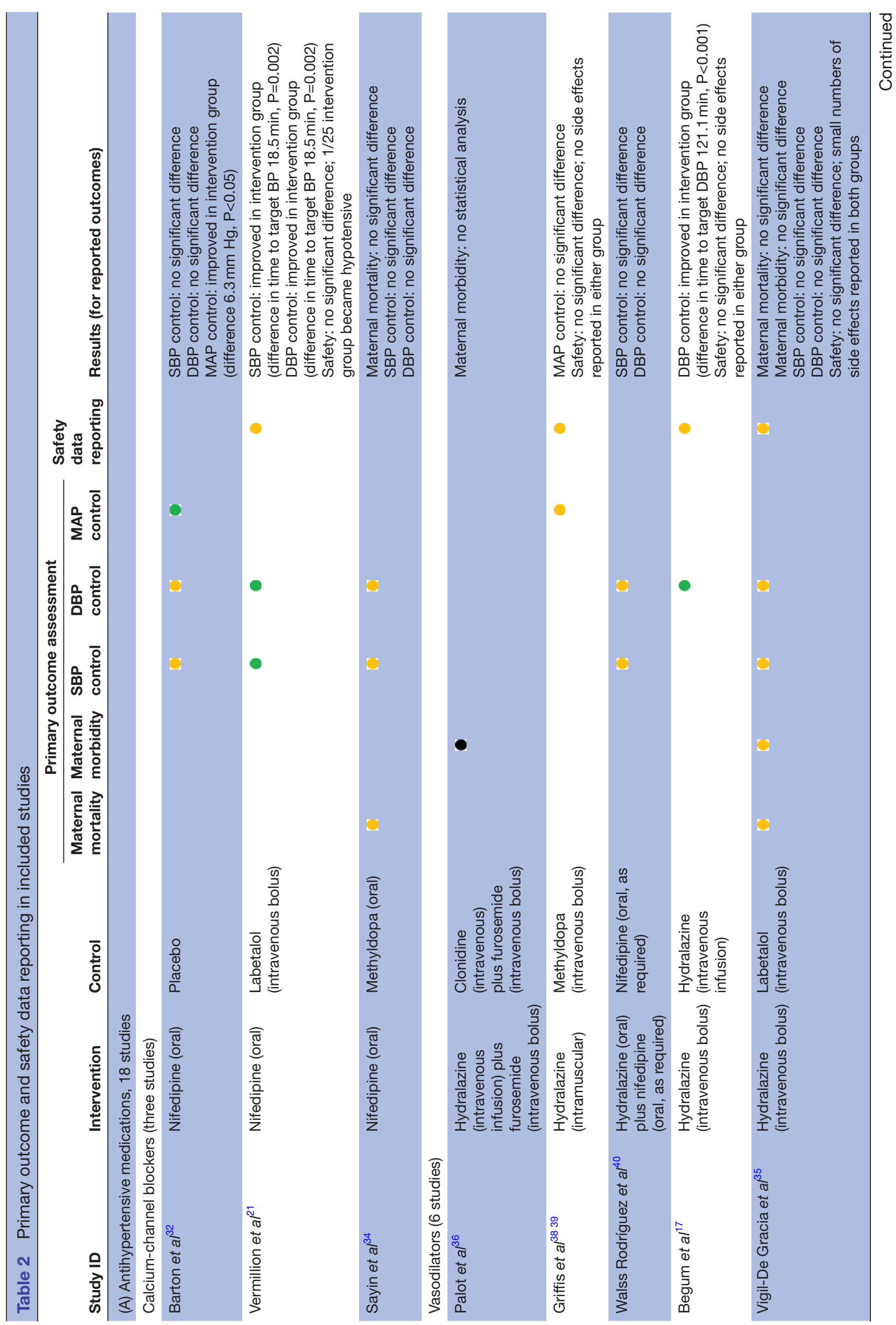




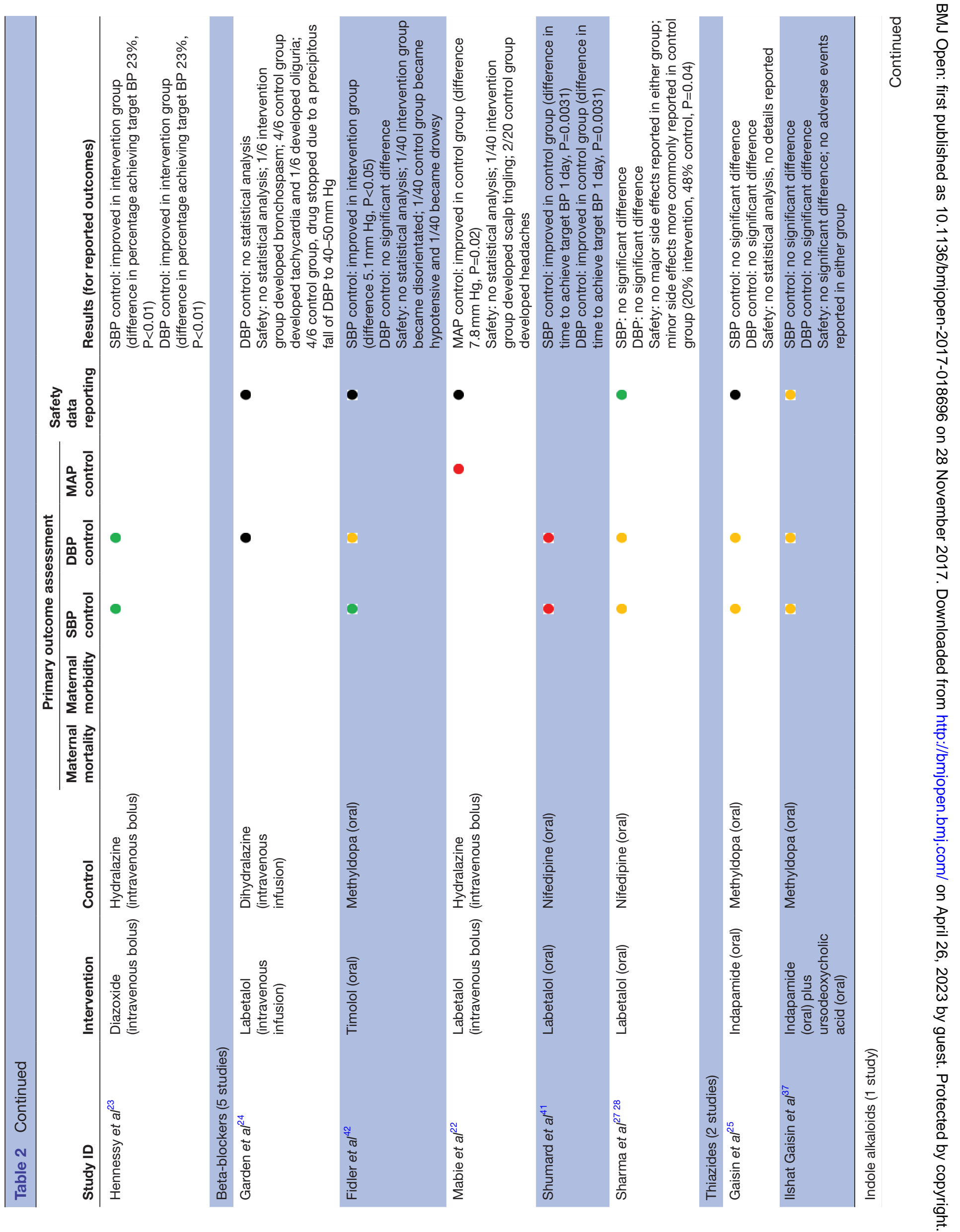



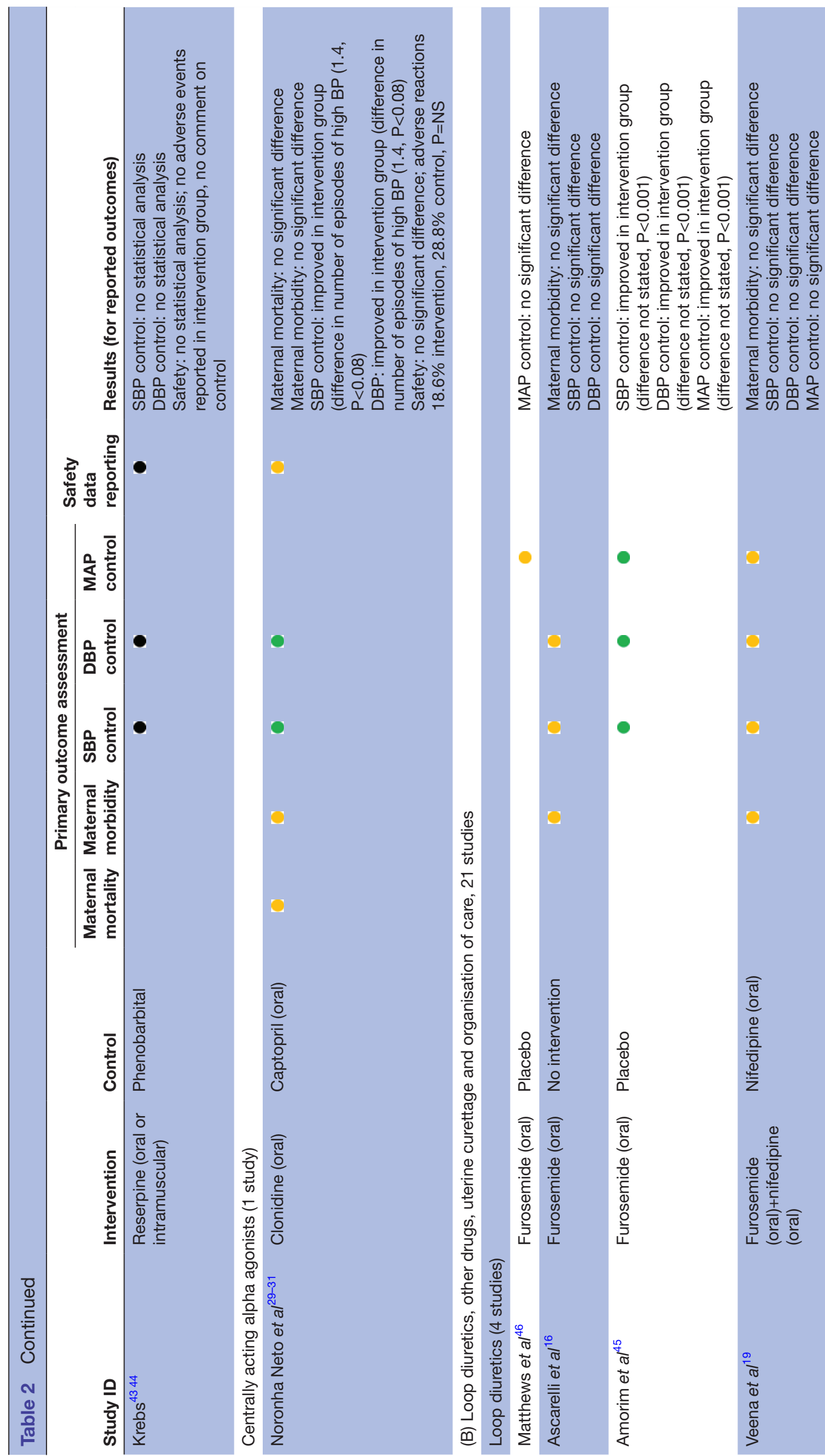


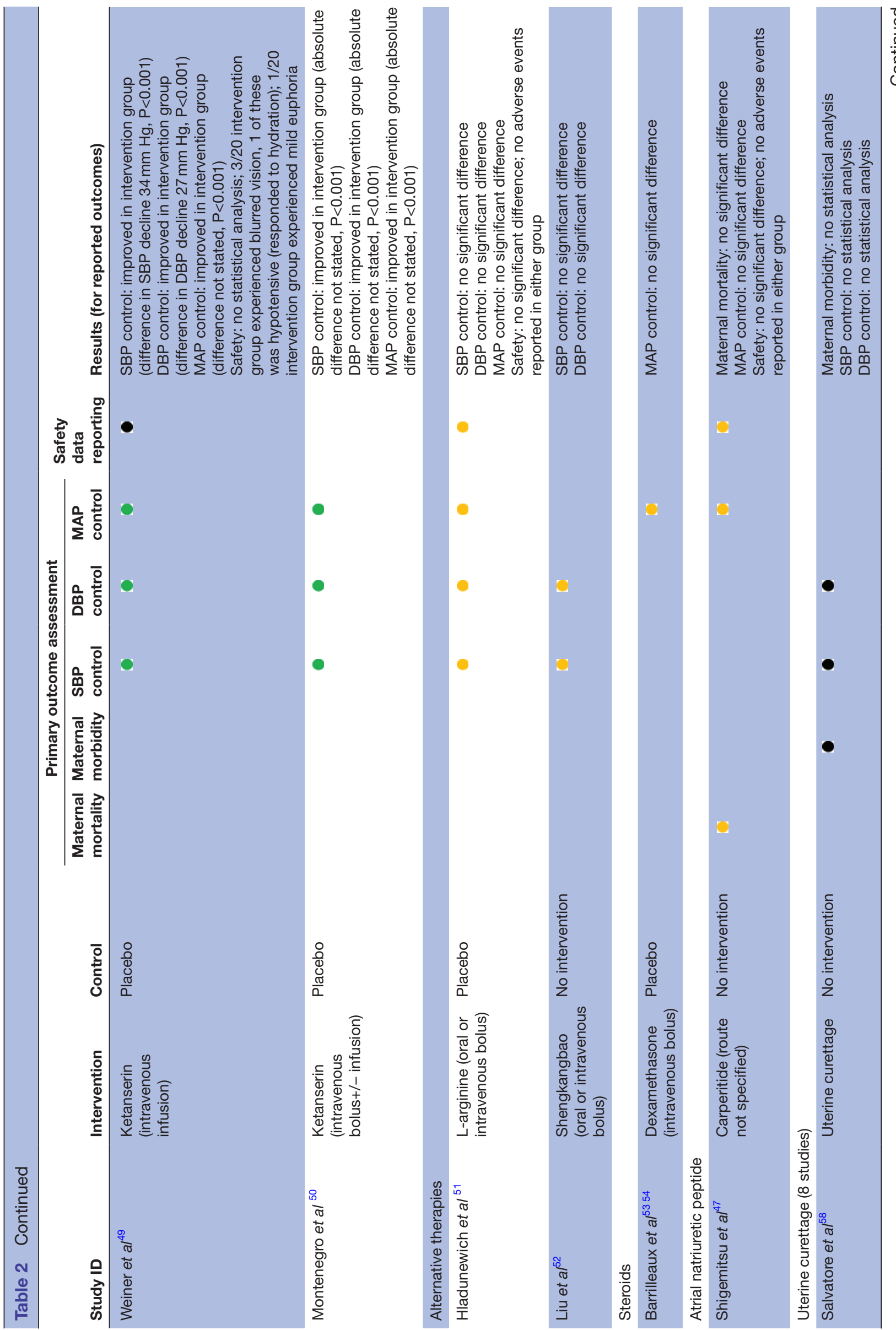

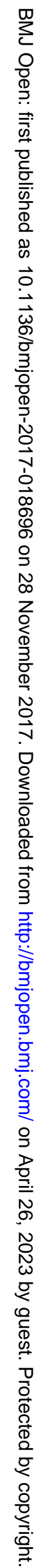




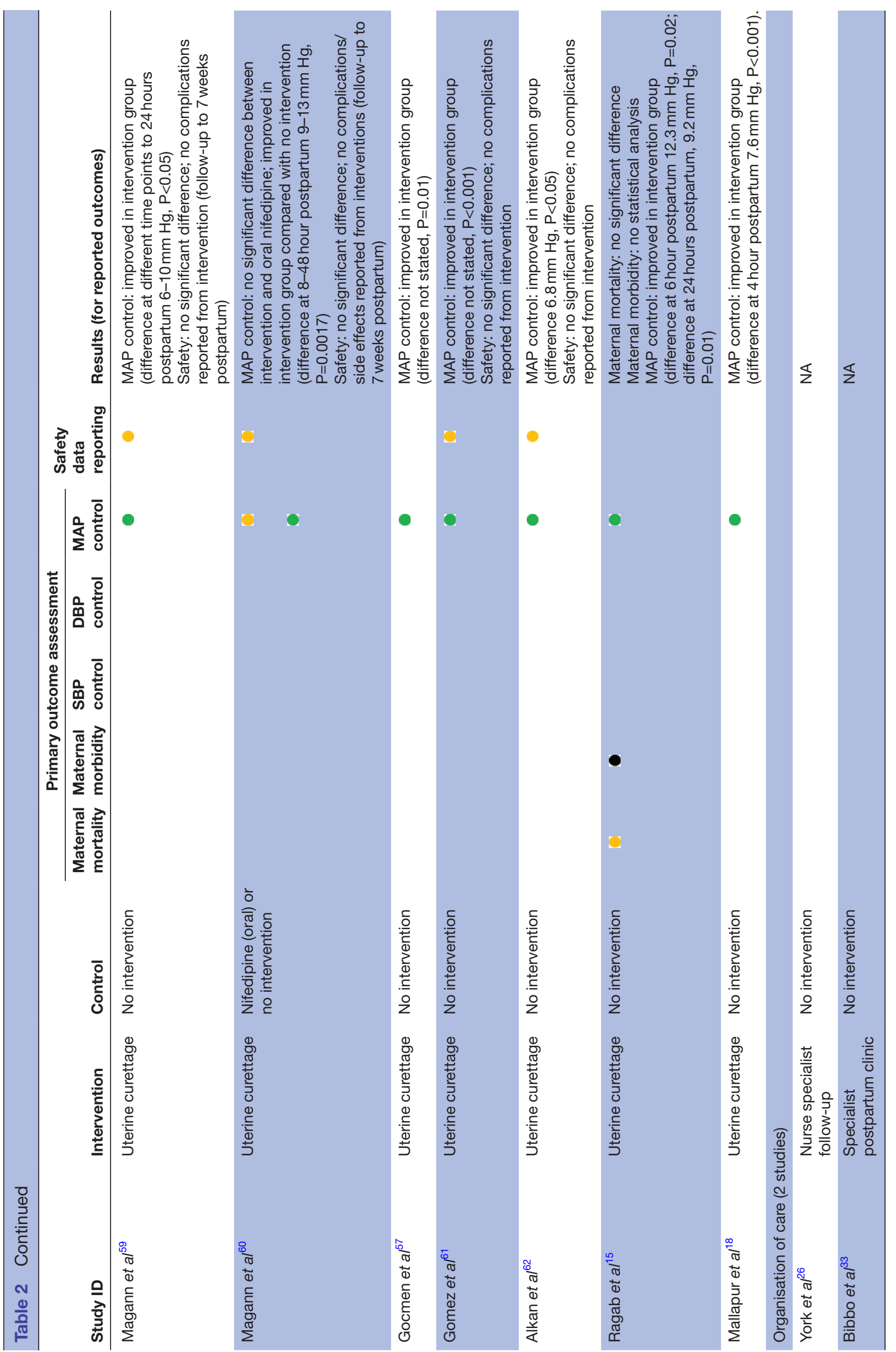


to 24 hours (one RCT, $\mathrm{n}=26$ ). ${ }^{38}{ }^{39}$ There was no difference in $\mathrm{BP}$ control when comparing oral hydralazine with oral nifedipine (one RCT, $\mathrm{n}=38$ ) or intravenous labetalol (one RCT, $\mathrm{n}=82) .{ }^{35} 40$

Bolus diazoxide was significantly more effective in achieving a target $\mathrm{BP}$ of $\leq 140 / 90 \mathrm{~mm} \mathrm{Hg}$ than intravenous hydralazine (intervention group 67\%, control group $43 \%$; relative risk (RR) $0.64,95 \%$ CI 0.46 to 0.89 ; one RCT, n=37 (postnatal)). ${ }^{23}$ One retrospective cohort study did not present any statistical analysis. ${ }^{36}$

\section{Beta-blockers}

Five studies assessed the efficacy of beta-blockers (four RCTs and one retrospective cohort study, $n=305$ ). Two RCTs compared intravenous labetalol with intravenous hydralazine/dihydralazine; one involved only six postnatal women and presented no statistical analysis of the data. ${ }^{24}$ The other found a significantly greater mean maximal decrease in MAP with intravenous labetalol (intervention group $25.5 \pm 11.2 \mathrm{~mm} \mathrm{Hg}$, control group $33.3 \pm 13.2 \mathrm{~mm} \mathrm{Hg}$ : difference $-7.8 \mathrm{~mm} \mathrm{Hg}, \mathrm{P}=0.02$; one RCT, n=32 (postnatal)). ${ }^{22}$ Results conflicted regarding whether oral labetalol was more or less effective than oral nifedipine; a cohort study reported that labetalol controlled BP less rapidly than nifedipine (intervention group 2.7 days, control group 1.7 days: difference 1.0 days, $\mathrm{P}=0.0031$; one retrospective cohort study, $\mathrm{n}=128){ }^{41}$ However, this result was not replicated by an RCT, where the time to BP control was similar in the two groups $(\mathrm{n}=50) .{ }^{27}{ }^{28}$ Neither study demonstrated a difference in the postnatal length of stay $(n=178)$. Timolol was effective in decreasing diastolic $\mathrm{BP}$ on the first day postnatal when compared with methyldopa (intervention group $88.7 \mathrm{~mm} \mathrm{Hg}$, control group $93.8 \mathrm{~mm} \mathrm{Hg:} \mathrm{difference}$ $-5.1 \mathrm{~mm} \mathrm{Hg}, \mathrm{P}<0.05$; one RCT, $\mathrm{n}=80) .{ }^{42}$

\section{Other antihypertensive medications}

No statistically significant difference was found between oral clonidine and oral captopril in the incidence of episodes of severe hypertension postpartum (one RCT, $\mathrm{n}=90) \cdot{ }^{29-31}$ Two RCTs evaluating indapamide versus methyldopa found no difference in BP control over 6-12 months postpartum $(\mathrm{n}=60) .{ }^{25}{ }^{37}$ One retrospective cohort study $(n=140)$ compared reserpine with phenobarbital; the results suggested that reserpine might achieve faster and greater BP reduction (data extracted from graphs; no statistical analysis). No adverse events were reported in the intervention group. ${ }^{43} 44$

\section{What are the benefits and harms of other therapeutic interventions for women with HDP postpartum? Loop diuretics}

Four RCTs ( $\mathrm{n}=503$ ) examined loop diuretics versus placebo or usual care in postpartum hypertension management in women with HDP. None reported maternal mortality or safety data. Only two reported major maternal morbidity, neither demonstrating a difference between groups (table 2B). ${ }^{1619}$
One RCT ( $n=120)$ reported significant improvement in the primary outcome of mean systolic and diastolic BP with oral furosemide versus placebo (magnitude of difference or time points of measurements not stated, $\mathrm{P}<0.001) .{ }^{45}$ This was not the case in the other placebo-controlled RCT, which found no significant difference $(n=19) .{ }^{46}$ Two further RCTs $(n=364)$ found no significant difference in BP control with oral furosemide versus usual care. ${ }^{16} 19$ In one of these, subgroup analysis of women with severe pre-eclampsia $(n=70)$ found women who received oral furosemide had a significantly lower systolic BP at day 2 postpartum (intervention group $142 \pm 13 \mathrm{~mm} \mathrm{Hg}$, control group $153 \pm 19 \mathrm{~mm} \mathrm{Hg}$ : difference $-11 \mathrm{~mm} \mathrm{Hg}, \mathrm{P}<0.004)$, but not at other time points. ${ }^{16}$ In the other trial $(n=100)$, furosemide reduced the need for additional antihypertensive treatment during the 3 days of therapy (intervention group $8.0 \%$, control group $26.0 \%$ : difference $18 \%$, $\mathrm{P}=0.017$ ), but this difference did not persist to hospital discharge. ${ }^{19}$

\section{Other drugs}

Five RCTs, one quasi-randomised study and one retrospective cohort study investigated the utility of different drug classes in HDP postpartum (online supplementary appendix S5). Three studies reported safety data, but only one reported maternal mortality, demonstrating no difference between groups, ${ }^{47}$ and none reported major maternal morbidity (table $2 \mathrm{~B}$ ).

Three small, crossover RCTs examined the use of selective serotonin receptor inhibitors (SSRIs) compared with placebo $(n=55)$. All studies showed a significant reduction in BP with SSRIs compared with placebo (range 25.6-34 mm Hg). ${ }^{48-50}$ These data suggest efficacy for this drug class in hypertension management but do not provide any information regarding relative effectiveness compared with standard antihypertensive drugs. Only one study reported safety data; although no statistical analysis was performed, there were a number of side effects reported in the intervention group. ${ }^{49}$

Two studies evaluated alternative therapies $(n=117)$; there was no difference in BP control with L-arginine supplementation compared with placebo (one RCT, $\mathrm{n}=45) .{ }^{51}$ One reported accelerated recovery of albuminuria with the administration of shengkangbao (Chinese herbal medicine) versus placebo (one quasi-randomised study, $n=72$ ). However, the clinical relevance of this outcome is uncertain; there was no difference between groups in the secondary outcomes of systolic BP, diastolic $\mathrm{BP}$ or serum creatinine, and no safety data were reported. ${ }^{52}$

A single RCT assessed corticosteroids in the management of severe pre-eclampsia postpartum $(n=157){ }^{5354}$ No difference was demonstrated between groups in the primary outcome of antihypertensive medication requirement, or in the secondary outcomes of MAP or need for critical care admission, and no safety data were reported. There were small, statistically significant differences found in some laboratory values (platelet count, lactate dehydrogenase and aspartate transaminase). However, 
the authors acknowledged that the absolute differences were too small to be clinically relevant. ${ }^{53}$

A very small retrospective cohort study suggested an improvement in MAP with the addition of carperitide (atrial natriuretic peptide) to standard therapy $(n=16)$, and no adverse effects related to the intervention were reported. ${ }^{47}$ However, the magnitude of the difference was not published, and the study was too small to draw any firm conclusions.

\section{Uterine curettage}

Six RCTs and two prospective cohort studies $(n=837)$ have explored the role of uterine curettage in postpartum hypertension management. Uterine curettage is a similar process to that used in the surgical management of miscarriage; the lining of the uterus is scraped after completion of the third stage of labour in order to maximise placental tissue removal. This may be under direct vision following caesarean section, or via the transcervical route following vaginal birth. The latter approach may be ultrasound-guided and necessitates some form of anaesthesia. The theory underlying this intervention is that gestational hypertension and pre-eclampsia are placenta-mediated, and therefore ensuring complete evacuation of the uterus following childbirth may accelerate recovery. ${ }^{55} 56$

Seven studies explicitly stated they included both participants who delivered vaginally and those delivered by caesarean; four reported numbers undergoing vaginal delivery $(n=248)$ and caesarean $(n=321)$. One made no comment about the mode of birth. ${ }^{57}$ Only one study reported maternal mortality, and there was no difference between groups. ${ }^{15}$ Two reported major maternal morbidity, but neither performed any statistical analysis (table 2B). However, both studies did suggest a reduction in the absolute number of eclamptic seizures in the curettage group compared with no intervention. ${ }^{15} 58$ In one, however, there was a relevant difference between the study groups; $28 / 28(100 \%)$ in the control group were eclamptic at enrolment, compared with $9 / 20(45 \%)$ in the intervention group. ${ }^{58}$ Four studies reported safety data, with none reporting any complications related to the intervention (table 2B). ${ }^{59-62}$

All eight studies compared curettage with standard care (ie, no additional intervention), and all suggested that uterine curettage resulted in a significantly lower BP. ${ }^{15} 18$ 57-62 One of these had two control groups: standard care and oral nifedipine; when compared with oral nifedipine, no difference was noted with curettage. ${ }^{60}$

Five studies reported the magnitude of the difference in MAP between curettage and standard care: range 6-13 mm Hg. ${ }^{15} 18596062$ Only two of these reported BP data beyond 24 hours postpartum; one RCT reported a significantly lower MAP at 48 hours with curettage (intervention group $104 \mathrm{~mm} \mathrm{Hg}$, control group $113 \mathrm{~mm} \mathrm{Hg:}$ difference $9 \mathrm{~mm} \mathrm{Hg}, \mathrm{P}=0.0017 ; \mathrm{n}=45),{ }^{60}$ but the other RCT demonstrated no significant difference in MAP at 48 hours $(n=420) .{ }^{15}$
One study demonstrated that a greater proportion of the intervention group attained the target BP of $<140 / 90 \mathrm{~mm} \mathrm{Hg}$ at 24 (intervention group 9/20 (45\%), control group $3 / 28$ (11\%): difference $34 \%$, no $\mathrm{P}$ value quoted) and 48 hours postpartum (intervention group $14 / 20(70 \%)$, control group 8/28 (29\%): difference $41 \%$, no $\mathrm{P}$ value quoted) ${ }^{58}$ Two studies did not present the size of the difference between groups. ${ }^{5761}$

\section{DISCUSSION}

This review found evidence demonstrating that calcium-channel blockers, vasodilators and beta-blockers lower BP postpartum, but no clear answer to which was most effective and should, therefore, be preferentially prescribed. All but two studies examined the acute control of severe hypertension or short-term BP control while women remained in hospital postpartum, ${ }^{25} 37$ and so provide little guidance about prescription in the weeks after discharge. Moreover these both examined thiazide diuretics, not recommended in the UK for use while breast feeding. ${ }^{8}$ Complete safety data were limited across trials, as were data regarding objective clinical outcomes, and two further studies examined antihypertensive agents not recommended for use postpartum in the UK (methyldopa and reserpine). ${ }^{6364}$ One trial evaluated captopril at a much higher daily dose than the UK recommended daily starting dose. ${ }^{64}$

Uterine curettage is not currently recommended, due to safety concerns regarding additional anaesthetic and operative risks, and the availability of alternative treatments to lower BP, particularly in the context of vaginal birth. ${ }^{65}$ However, the included studies consistently demonstrated that uterine curettage improved BP control versus standard care,,$^{15} 8^{57-62}$ with one reporting an equivalent effect to oral nifedipine. ${ }^{60}$ Among the limited safety data, none reported an excess complication rate (infection or uterine damage) with curettage, but given the low incidence of operative complications, the total population $(\mathrm{n}=837)$ was likely insufficient to adequately address potential competing risks. Furthermore, these studies did not demonstrate any impact from curettage on maternal mortality or severe morbidity, and concerns exist about some studies' methodology. The evidence reviewed is insufficient to recommend incorporation of this intervention into routine clinical practice.

Four trials evaluating loop diuretics failed to provide conclusive evidence of benefit. Three produced non-significant results in their main analysis, ${ }^{16} 1946$ and the single conference abstract, which did suggest better BP control with oral furosemide, did not publish the magnitude of the difference, rendering it difficult to assess the clinical relevance. ${ }^{45}$ In contrast to the Cochrane review, we conclude that, at present, there is no evidence to support the routine use of diuretics postpartum. ${ }^{10}$

We found no adequate evidence to support alternative medications or a particular care model in the management of HDP postpartum. SSRIs substantially reduced BP 
versus placebo, ${ }^{48-50}$ but no published data were identified comparing their efficacy with standard antihypertensive treatment, making it difficult to draw meaningful conclusions about their clinical application. Neither study evaluating postpartum care organisation reported maternal mortality or morbidity, or any measure of BP control, with both selecting postnatal readmissions as their primary outcome. An increased postnatal readmission rate, however, may not necessarily reflect harm; it might instead suggest that a particular model of care can better detect problems in the community and admit appropriately, ultimately resulting in a lower risk to patients.

In light of the heterogeneous nature of research in this field, when designing this review, we included all interventions targeting hypertension management, but not end-organ complications, including eclampsia. Therefore, trials evaluating magnesium sulfate were outside the scope of this review. We acknowledge the relevance of this therapy in women with severe pre-eclampsia, especially in the immediate postnatal period, and a Cochrane review suggests there is no uncertainty regarding the effectiveness of this therapy. ${ }^{66}$

A strength of this review is that cohort studies, casecontrol studies and quasi-randomised studies were eligible in addition to RCTs, and no language or date restrictions were imposed, resulting in a comprehensive review that provides evidence suggesting significant research gaps, consistent with the findings from the Cochrane review (2013). ${ }^{10}$ The applicability of the findings and recommendations from this review is restricted by the low quality of included studies; both reviewers judged the vast majority to be at high overall risk of bias. Nearly a quarter of the included studies were published only as conference abstracts, and therefore not subjected to peer review. Data extraction was restricted to the information provided in the abstracts (no authors provided additional data on request). These were limiting factors in our analysis, but we nonetheless felt it was important to include these studies for completeness, especially given the paucity of evidence that exists in this field. A further justification for their inclusion is that half of the trials reported in conference abstracts never reach full publication, and positive trials are more likely to be published than negative ones, ${ }^{67}$ which has the potential to skew the results of a review if they are omitted.

A further limitation of this review is that the majority of identified studies did not report substantive clinical outcomes such as maternal mortality, morbidity or harms. Without these, it is difficult to define properly the potential role of proposed interventions in clinical practice. The incidence of adverse maternal and neonatal outcomes, particularly in high-resource settings, is low, meaning adequately powering studies for real outcomes of interest is financially demanding. Therefore researchers often employ surrogate outcomes. Additionally, the range of outcomes reported in included studies was broad and inconsistent, with BP changes in particular being measured in a variety of different ways, further limiting the comparability of trials. Increasingly, core outcome sets are being produced, with a view to trials reporting as standard a minimum set of outcomes that are clinically meaningful and important to patients. ${ }^{68}$ We hope in the future that this would enhance our ability to synthesise results from different studies to produce high-quality evidence. There is consensus about trying to move away from surrogate outcomes, for example time to BP control, as they cannot effectively substitute for clinically important outcomes. An important and clinically meaningful end point should measure how a patient feels, functions or survives .

The Cochrane review included only nine randomised trials (author names in bold in online supplementary appendix S4). We believe our review adds to this, as an additional 30 studies are included (19 predating the Cochrane search, and 11 subsequent to it), providing a current and complete summary of all available research in the field. The contrast between the scales of the two reviews highlights a lack of high-quality evidence, despite a reasonably high number of research studies being conducted to answer the question about how hypertension should be managed postpartum in women with HDP. In future, studies need to be more robust and better designed to address the research questions adequately. Furthermore, in spite of these extensions, the body of evidence identified was substantially smaller than that underpinning antenatal hypertension management; 18 studies $(n=982)$, not restricted to RCTs, evaluated antihypertensive medications postpartum. Furthermore, the size of all but a few individual studies was small. In comparison, a Cochrane review (2014) evaluating antihypertensive medication for mild to moderate hypertension in pregnancy included 49 RCTs $(n=4723) .{ }^{69}$ Moreover, the quantity and quality of evidence supporting the management of HDP are vastly less than that available for essential hypertension outside pregnancy, where individual RCTs commonly involve several thousand participants. ${ }^{70}$

This review demonstrates a lack of good-quality evidence for postpartum hypertension management, emphasising the need for further RCTs directly comparing different antihypertensive agents, BP thresholds for medication adjustment and different models of care, with outcome measures other than postnatal readmissions. We believe the studies examining uterine curettage justify further research to evaluate clinically meaningful outcomes and procedural risks. It might be pragmatic to confine this to curettage at caesarean section, given concerns regarding surgical intervention after vaginal birth; an additional anaesthetic is not required; infection risk is lowered within a sterile surgical field compared with the transcervical route; and curettage under direct vision limits perforation risk. This might be beneficial in women with severe pre-eclampsia, where BP control during pregnancy has been challenging despite multiple medications. ${ }^{55}$

Acknowledgements The authors would like to thank Dr Ly-Mee Yu, Dr Helen Cotton and Dr Victoria E Cairns for their assistance with translation. 
Contributors AEC drafted the protocol with JMND, and drafted and piloted the data extraction sheet. These were reviewed by RJMcM, LP, KLT, LHM and PL. NR and AEC wrote the search strategy, and the online searches were conducted by NR. AEC and LP reviewed the search results independently and carried out the data extraction. This manuscript was drafted by AEC and reviewed by RJMcM, JMND, LP, NR, KLT, LHM and PL. AEC will be the guarantor.

Funding The research was funded by the National Institute for Health Research (NIHR) Collaboration for Leadership in Applied Health Research and Care Oxford at Oxford Health NHS Foundation Trust, and via a Research Professorship awarded to RJMcM (NIHR-RP-02-12-015). The views expressed are those of the author(s) and not necessarily those of the NHS, the NIHR or the Department of Health.

\section{Competing interests None declared.}

Provenance and peer review Not commissioned; externally peer reviewed.

Data sharing statement Extra data can be accessed via the Dryad data repository at http://datadryad.org/ with the doi:10.5061/dryad.pb6f2.

Open Access This is an Open Access article distributed in accordance with the terms of the Creative Commons Attribution (CC BY 4.0) license, which permits others to distribute, remix, adapt and build upon this work, for commercial use, provided the original work is properly cited. See: http://creativecommons.org/ licenses/by/4.0/

(c) Article author(s) (or their employer(s) unless otherwise stated in the text of the article) 2017. All rights reserved. No commercial use is permitted unless otherwise expressly granted.

\section{REFERENCES}

1. Podymow T, August P. Postpartum course of gestational hypertension and preeclampsia. Hypertens Pregnancy 2010;29:294-300.

2. Goel A, Maski MR, Bajracharya S, et al. Epidemiology and mechanisms of De Novo and persistent hypertension in the postpartum period. Circulation 2015;132:1726-33.

3. Chames MC, Livingston JC, Ivester TS, et al. Late postpartum eclampsia: a preventable disease? Am J Obstet Gynecol 2002;186:1174-7.

4. Kayem G, Kurinczuk JJ, Spark P, et al. Maternal and obstetric factors associated with delayed postpartum eclampsia: a national study population. Acta Obstet Gynecol Scand 2011;90:1017-23.

5. Watson DL, Sibai BM, Shaver DC, et al. Late postpartum eclampsia: an update. South Med J 1983;76:1487-9.

6. Martin JN, Thigpen BD, Moore RC, et al. Stroke and severe preeclampsia and eclampsia: a paradigm shift focusing on systolic blood pressure. Obstet Gynecol 2005;105:246-54.

7. Berks D, Steegers EA, Molas M, et al. Resolution of hypertension and proteinuria after preeclampsia. Obstet Gynecol 2009;114:1307-14.

8. National Institute for Health and Care Excellence. NICE clinical guideline 107: hypertension in pregnancy: the management of hypertensive disorders during pregnancy, 2011.

9. ACOG Task Force on Hypertension in Pregnancy. Hypertension in pregnancy: American college of obstetricians and gynecologists, 2013.

10. Magee L, von Dadelszen P. Prevention and treatment of postpartum hypertension. Cochrane Database Syst Rev 2013;4.

11. Moher D, Shamseer L, Clarke M, et al. Preferred reporting items for systematic review and meta-analysis protocols (PRISMA-P) 2015 statement. Syst Rev 2015:4:1.

12. McManus RJ, Wilson S, Delaney BC, et al. Review of the usefulness of contacting other experts when conducting a literature search for systematic reviews. BMJ 1998;317:1562-3.

13. Higgins JP, Altman DG, Gøtzsche PC, et al. The Cochrane Collaboration's tool for assessing risk of bias in randomised trials. BMJ 2011;343:d5928.

14. Wells GA, Shea B, O'Connell D, et al. The Newcastle-Ottawa Scale (NOS) for assessing the quality of nonrandomised studies in metaanalyses. 2017 http://www.ohri.ca/programs/clinical_epidemiology/ oxford.htm (accessed 14 Jul 2017).

15. Ragab A, Goda H, Raghib M, et al. Does immediate postpartum curettage of the endometrium accelerate recovery from preeclampsia-eclampsia? A randomized controlled trial. Arch Gynecol Obstet 2013;288:1035-8.

16. Ascarelli MH, Johnson V, McCreary H, et al. Postpartum preeclampsia management with furosemide: a randomized clinical trial. Obstet Gynecol 2005;105:29-33.
17. Begum MR, Quadir E, Begum A, et al. Management of hypertensive emergencies of pregnancy by hydralazine bolus injection vs continuous drip-a comparative study. Medscape Womens Health 2002;7:1.

18. Mallapur A, Renuka B, Katageri G, et al. Role of postpartum curettage in recovery of severe preeclampsia and eclampsia patients-a randomised controlled trial. Int J Gynaecol Obstet 2015;131:E191.

19. Veena P, Perivela L, Raghavan SS. Furosemide in postpartum management of severe preeclampsia: a randomized controlled trial. Hypertens Pregnancy 2017;36:84-9.

20. United Nations Department of Economic and Social Affairs. World economic situation and prospects. New York, United States of America: United Nations, 2016.

21. Vermillion ST, Scardo JA, Newman RB, et al. A randomized, doubleblind trial of oral nifedipine and intravenous labetalol in hypertensive emergencies of pregnancy. Am J Obstet Gynecol 1999;181:858-61.

22. Mabie WC, Gonzalez AR, Sibai BM, et al. A comparative trial of labetalol and hydralazine in the acute management of severe hypertension complicating pregnancy. Obstet Gynecol 1987;70:328-33

23. Hennessy A, Thornton CE, Makris A, et al. A randomised comparison of hydralazine and mini-bolus diazoxide for hypertensive emergencies in pregnancy: the PIVOT trial. Aust N Z J Obstet Gynaecol 2007;47:279-85.

24. Garden A, Davey DA, Dommisse J. Intravenous labetalol and intravenous dihydralazine in severe hypertension in pregnancy. Clin Exp Hypertens $B$ 1982;1:371-83.

25. Gaisin IR, Iskchakova AS, Shilina LV. Indapamide in the management of post-partum hypertension: a randomized, case-control study. Eur Heart J 2013;34:P1426.

26. York R, Brown LP, Samuels $\mathrm{P}$, et al. A randomized trial of early discharge and nurse specialist transitional follow-up care of high-risk childbearing women. Nurs Res 1997;46:254-61.

27. Sharma KJ, Greene N, Kilpatrick SJ. 40: Oral labetalol compared to oral extended release nifedipine for persistent postpartum hypertension: a randomized controlled trial. Am J Obstet Gynecol 2016;214:S27-8.

28. Sharma KJ, Greene N, Kilpatrick SJ. Oral labetalol compared to oral nifedipine for postpartum hypertension: a randomized controlled trial. Hypertens Pregnancy 2017;36:44-7.

29. Noronha Neto C C, Maia SS, Katz L, et al. Clonidine versus captopril for severe postpartum hypertension: a randomized controlled trial. PLoS One 2017;12:e0168124.

30. Amorim MMR, Noronha-Neto C, Maia SB, et al. Clonidine compared with captopril for severepostpartum hypertension. Obstet Gynecol 2015;125:42S

31. Katz L, Noronha Neto C, Maia S, et al. Clonidine versus captopril for severe postpartum hypertension: a randomized controlled trial. Pregnancy Hypertens 2015;5:29-30.

32. Barton JR, Hiett AK, Conover WB. The use of nifedipine during the postpartum period in patients with severe preeclampsia. Am J Obstet Gynecol 1990;162:788-92.

33. Bibbo C, Celi A, Thomas AM, et al. Does the addition of a specialized postpartum clinic improvethe care of women with preeclampsia? Obstet Gynecol 2014;123:39S.

34. Sayin NC, Altundag G, Varol FG. Efficacy of alpha-methyldopa and nifedipine in the treatment of postpartum hypertension. [Turkish] Postpartum hipertansiyon tedavisinde alfametildopa ve nifedipinin etkinligi. J Turk Ger Gynecol Assoc 2005;6:118-22.

35. Vigil-De Gracia P, Ruiz E, López JC, et al. Management of severe hypertension in the postpartum period with intravenous hydralazine or labetalol: a randomized clinical trial. Hypertens Pregnancy 2007;26:163-71

36. Palot M, Jakob L, Decaux J. Arterial hypertensions of labor and the post-partum period. [French] Les Hypertensions Arterielles Du Travail Et Du Post-Partum. Rev Fr Gynecol Obstet 1979;74:173-6.

37. Ilshat Gaisin IR, Iskchakova AS, Shilina LV. Control of cardiovascular risk factors with ursodeoxycholic acid and indapamide in postpreeclamptic nursing mothers: Results from a randomized, case-control 1-year study. Eur J Prev Cardiol 2014;21:S120

38. Griffis KR, Martin JN, Palmer SM, et al. Utilization of hydralazine or alpha-methyldopa for the management of early puerperal hypertension. Am J Perinatol 1989;6:437-41.

39. Martin JN, Griffis KR, Martin RW, et al. Early puerperal hypertension management: hydralazine vs methyldopa. Clin Exp Hypertens $B$ 1988;8:431

40. Walss Rodríguez RJ, Villarreal Ordaz F. [Management of severe pre-eclampsia in the puerperium. Comparative study of sublingual nifedipine and hydralazine]. Ginecol Obstet Mex 1991;59:207-10. 
41. Shumard K, Yoon J, Huang C, et al. 718: Peripartum antihypertensive choice affects time to blood pressure control in treating hypertensive disorders of pregnancy. Am J Obstet Gynecol 2016;214:S378.

42. Fidler J, Smith V, De Swiet M. A randomized study comparing timolol and methyldopa in hospital treatment of puerperal hypertension. $\mathrm{Br} \mathrm{J}$ Obstet Gynaecol 1982;89:1031-4.

43. Krebs A. [Experience with serpasil and luminal in the management of pregnancy-and puerperal toxemias]. Gynaecologia 1956;141:255-60.

44. Krebs A. [Tests of reserpine and phenobarbital in therapy of toxicosis of pregnancy and puerperium]. Geburtshilfe Frauenheilkd 1956;16:410-22.

45. Amorim M, Katz L, Cursino T, et al. Postpartum furosemide for accelerating recovery in women with severe preeclampsia: A randomized clinical trial. Int J Gynaecol Obstet 2015;131:E195.

46. Matthews G, Gornall R, Saunders NJ. A randomised placebo controlled trial of loop diuretics in moderate/severe pre-eclampsia, following delivery. J Obstet Gynaecol 1997;17:30-2.

47. Shigemitsu A, Akasaka J, Shigetomi H, et al. Use of carperitide for postpartum diuresis of severe preeclampsia. Pregnancy Hypertens 2015:5:145.

48. Weiner CP, Serotonin SML. 5HT) receptor blockade in puerperal preeclampsia. Clin Exp Hypertens B 1982:1:195.

49. Weiner CP, Socol ML, Vaisrub N. Control of preeclamptic hypertension by ketanserin, a new serotonin receptor antagonist. $A m$ J Obstet Gynecol 1984;149:496-500.

50. Montenegro R, Knuppel RA, Shah D, et al. The effect of serotonergic blockade in postpartum preeclamptic patients. Am J Obstet Gynecol 1985:153:130-3.

51. Hladunewich MA, Derby GC, Lafayette RA, et al. Effect of L-arginine therapy on the glomerular injury of preeclampsia: a randomized controlled trial. Obstet Gynecol 2006;107:886-95.

52. Liu Z, Wang XY, Yan NN. [Treatment of albuminuria in gestational hypertension puerpera in the severe preeclampeia stage by TCM therapy for stasis-removing and diuresis]. Zhongguo Zhong Xi Yi Jie He Za Zhi 2009;29:222-4.

53. Barrilleaux PS, Martin JN, Klauser CK, et al. Postpartum intravenous dexamethasone for severely preeclamptic patients without hemolysis, elevated liver enzymes, low platelets (HELLP) syndrome: a randomized trial. Obstet Gynecol 2005;105:843-8.

54. Barrilleaux PS, Martin J, Klauser C, et al. Adjunctive intravenous dexamethasone in patients with severe preeclampsia not complicated by HELLP syndrome. Am J Obstet Gynecol 2003;189:S94.

55. Caughey AB. Editorial Comment: Does Immediate Postpartum Curettage of the Endometrium Accelerate Recovery From
Preeclampsia-Eclampsia? A Randomized Controlled Trial. Obstet Gynecol Surv 2014;69:121-2.

56. HUNTER CA, Howard WF, McCORMICK CO. Amelioration of the hypertension of toxemia by postpartum curettage. Am J Obstet Gynecol 1961;81:884-9.

57. Gocmen A, Yayla M, Ceylan Erden A, et al. The effect of postpartum curettage in severe preeclampsia and eclampsia in recovery (Ağır preeklampsı ve eklampsi in iyileşmesinde postpartum kuretajin etkisi). Perinatoloji Dergisi 1996;4:24.

58. Salvatore CA, Carduz E, Ciccivizzo E, et al. ["Postpartum curettage in severe toxemia with and without premature loosening of the placenta"]. Matern Infanc 1967;26:275-86.

59. Magann EF, Martin JN, Isaacs JD, et al. Immediate postpartum curettage: accelerated recovery from severe preeclampsia. Obstet Gynecol 1993;81:502-6.

60. Magann EF, Bass JD, Chauhan SP, et al. Accelerated recovery from severe preeclampsia: uterine curettage versus nifedipine. J Soc Gynecol Investig 1994;1:210-4.

61. Gomez LM, De la Vega GA, Ludmir J, et al. Immediate postpartum curettage accelerates clinical recovery in severe preeclampsia. Obstet Gynecol 2005;105:111S.

62. Alkan A, Tugrul S, Oral O, et al. Effects of postpartum uterine curettage on maternal well-being in severe preeclamptic patients. Clin Exp Obstet Gynecol 2006;33:55-8.

63. Webster J, Koch HF. Aspects of tolerability of centrally acting antihypertensive drugs. J Cardiovasc Pharmacol 1996;27:S49-54.

64. Committee JF. British National Formulary (BNF). 71st edn. BMJ Publishing Group Ltd and Royal Pharmaceutical Society, 2016.

65. Tan LK, de Swiet M. The management of postpartum hypertension. BJOG 2002;109:733-6.

66. Duley L, Gulmezoglu AM, Henderson-Smart DJ, et al. Magnesium sulphate and other anticonvulsants for women with pre-eclampsia. Cochrane Database Syst Rev 2010;11.

67. Scherer RW, Langenberg P, von Elm E. Full publication of results initially presented in abstracts. Cochrane Database Syst Rev 2007;2:MR000005

68. Khan K. The CROWN Initiative: journal editors invite researchers to develop core outcomes in women's health. Br J Obstet Gynaecol 2014;121:1181-2.

69. Abalos E, Duley L, Steyn DW. Antihypertensive drug therapy for mild to moderate hypertension during pregnancy. Cochrane Database Syst Rev 2014;2.

70. National Institute for Health and Care Excellence. NICE clinical guideline 127: hypertension in adults: diagnosis and management, 2011. 